\title{
A FAREY TREE ORGANIZATION OF LOCKING REGIONS FOR SIMPLE CIRCLE MAPS
}

\author{
K. M. BRUCKS AND C. TRESSER \\ (Communicated by Linda Keen)
}

\begin{abstract}
Let $f$ be a $C^{3}$ circle endomorphism of degree one with exactly two critical points and negative Schwarzian derivative. Assume that there is no real number $a$ such that $f+a$ has a unique rotation number equal to $\frac{p}{q}$. Then the same holds true for any $\frac{p^{\prime}}{q^{\prime}}$ such that $\frac{p}{q}$ stands above $\frac{p^{\prime}}{q^{\prime}}$ in the Farey tree and can be related to it by a path on the tree.
\end{abstract}

\section{INTRODUCTION}

Let $F$ be a degree-one lift, i.e., $F: \mathbb{R} \rightarrow \mathbb{R}$ is a continuous map such that $F(x+1)=F(x)+1$ for all $x \in \mathbb{R}$. Hence, $F$ is a lift to the universal cover $\mathbb{R}$ of some degree-one endomorphism $f$ of the circle $\mathbf{T}=\mathbb{R} / \mathbb{Z}$, i.e., $\pi \circ F=f \circ \pi$, where $\pi: \mathbb{R} \rightarrow \mathbf{T}$ is given by $\pi(x)=x \bmod 1$. For each $x \in \mathbb{R}$ we set

$$
\underline{\rho}_{F}(x)=\liminf _{m \rightarrow \infty} \frac{F^{m}(x)-x}{m} \quad \text { and } \quad \bar{\rho}_{F}(x)=\limsup _{m \rightarrow \infty} \frac{F^{m}(x)-x}{m},
$$

where as usual $F^{0}=\mathrm{Id}$ and $F^{m}=F \circ F^{m-1}$. We define,

$$
\begin{aligned}
& \underline{\operatorname{Rot}}(F)=\left\{\underline{\rho}_{F}(x) \mid x \in \mathbb{R}\right\} \\
& \overline{\operatorname{Rot}}(F)=\left\{\bar{\rho}_{F}(x) \mid x \in \mathbb{R}\right\} \\
& \operatorname{Rot}(F)=\left\{\rho_{F}(x) \mid x \in \mathbb{R} \text { and } \rho_{F}(x)=\underline{\rho}_{F}(x)=\bar{\rho}_{F}(x)\right\}
\end{aligned}
$$

It is known that $\underline{\operatorname{Rot}}(F)=\overline{\operatorname{Rot}}(F)=\operatorname{Rot}(F)$ and that this set is either a single point or a non-degenerate closed interval (see e.g. [1], Theorem 3.7.20). Throughout this note we make many references to [1] both for the convenience of the reader and of the authors; the reader will find original references traced and discussed in [1]. The map $F$ is said to be frequency locked if $\operatorname{Rot}(F)$ is a single point. Any degreeone lift which is non-decreasing is frequency locked; this is a simple extension of the classical theory of rotation numbers developed by Poincaré [12]. Thus for such non-decreasing degree-one lifts, the above limsup and liminf can be replaced by a true lim symbol and there is no $x$-dependence.

As described for instance in [3] and in [11], the characterization of frequencylocked maps which are not necessarily injective is simplest when the maps are

Received by the editors July 20, 1994.

1991 Mathematics Subject Classification. Primary 58F03. 
$C^{3}$, have at most two critical points, and their Schwarzian derivative is negative (where defined). Hence, we say a degree-one lift $F$ belongs to Class $\mathcal{A}$ if $F$ is strictly piecewise monotone with precisely two turning points in the interval $(0,1)$, a maximum $C$ and a minimum $K$ with $K>C$; when more than one lift is used we write $K_{F}$ and $C_{F}$ to avoid confusion (see Figure 4 ). If in addition, $F$ has a negative Schwarzian derivative (where defined), then we say $F$ belongs to Class $\mathcal{B}$. Our main result, Theorem 1 , is given for maps in Class $\mathcal{B}$; we remark that our main result also holds for piecewise affine maps with two turning points per period (these maps are described in [14]). Recall that the Schwarzian derivative of $F$ is defined as

$$
S(F)=\frac{F^{\prime \prime \prime}}{F^{\prime}}-\frac{3}{2}\left(\frac{F^{\prime \prime}}{F^{\prime}}\right)^{2} .
$$

If $F$ is any degree-one lift, we can construct a one-parameter family of degree-one lifts from $F$ by setting $F_{a}(x)=F(x)+a$ for $a \in \mathbb{R}$.

Now we pass to some definitions from number theory. Throughout this note all fractions are in lowest terms unless otherwise mentioned. The fractions $\frac{p_{1}}{q_{1}}$ and $\frac{p_{2}}{q_{2}}$ are said to be Farey neighbors if they satisfy the identity $p_{1} q_{2}-p_{2} q_{1}= \pm 1$. An arbitrary pair of fractions $\frac{p_{1}}{q_{1}}$ and $\frac{p_{2}}{q_{2}}$ can be combined to form their mediant, defined as $\frac{p_{1}+p_{2}}{q_{1}+q_{2}}$, which is not necessarily in lowest terms. In case $\frac{p_{1}}{q_{1}}$ and $\frac{p_{2}}{q_{2}}$ are Farey neighbors, $\frac{p_{1}}{q_{1}} \oplus \frac{p_{2}}{q_{2}} \stackrel{\text { def }}{=} \frac{p_{1}+p_{2}}{q_{1}+q_{2}}$ is necessarily in lowest terms and is called their Farey sum.

Proposition $1[8,9]$. Every rational number $\frac{p}{q} \in(0,1)$ can be expressed uniquely as a Farey sum $\frac{p}{q}=\frac{p_{1}}{q_{1}} \oplus \frac{p_{2}}{q_{2}}$.

The fractions $\frac{p_{1}}{q_{1}}$ and $\frac{p_{2}}{q_{2}}$ are the (Farey) parents of $\frac{p}{q}$. If $\frac{p_{1}}{q_{1}}<\frac{p_{2}}{q_{2}}$, then $\frac{p_{1}}{q_{1}}=L\left(\frac{p}{q}\right)$ is the left parent and $\frac{p_{2}}{q_{2}}=R\left(\frac{p}{q}\right)$ is the right parent. The children of $\frac{p}{q} \in(0,1)$ are the left child and right child defined respectively as $l\left(\frac{p}{q}\right)=L\left(\frac{p}{q}\right) \oplus \frac{p}{q}$ and $r\left(\frac{p}{q}\right)=\frac{p}{q} \oplus R\left(\frac{p}{q}\right)$. Finally we define one child for each of 0 and 1 by $r\left(\frac{0}{1}\right)=l\left(\frac{1}{1}\right)=\frac{1}{2}$. Throughout this note, for $\frac{p}{q} \in(0,1)$ we let $\frac{p_{1}}{q_{1}}<\frac{p_{2}}{q_{2}}$ denote the Farey parents of $\frac{p}{q}$.

The Farey tree FT is the infinite tree whose vertices are the set of rational numbers in $[0,1]$ with one edge between every fraction $\frac{p}{q}$ and each of its children (see Figure 1). The vertices fall into levels indexed by the non-negative integers. Vertices $\frac{0}{1}$ and $\frac{1}{1}$ are at level 0 , while the vertices at level $n>0$ are the children of the vertices at level $n-1$. For example, $\frac{2}{5}$ is at level $3, \frac{3}{7}$ is at level $4, \frac{1}{4}$ and $\frac{2}{5}$ are children of $\frac{1}{3}$, and $\frac{4}{7}$ and $\frac{5}{8}$ are children of $\frac{3}{5}$. Given two integers $m<n$ and two fractions on the tree, $\frac{p_{m}}{q_{m}}$ at level $m$ and $\frac{p_{n}}{q_{n}}$ at level $\mathrm{n}$, we say that $\frac{p_{m}}{q_{m}}$ is higher than $\frac{p_{n}}{q_{n}}$ on the Farey tree if these numbers are joined by a path on FT. For example, $\frac{1}{3}$ is higher than $\frac{3}{8}$, but not higher than $\frac{4}{7}$ (as there is no path between $\frac{1}{3}$ and $\frac{4}{7}$ ). We can now state our main result.

Theorem 1. Let $F$ be a degree-one lift in Class $\mathcal{B}$, and let $\left\{F_{a} \mid a \in \mathbb{R}\right\}$ be the one-parameter family defined by $F$. Let $\frac{p}{q}$ be higher than $\frac{p^{\prime}}{q^{\prime}}$ on the Farey tree. Then if no $F_{a}$ is frequency locked with frequency $\frac{p}{q}$, the same holds true for $\frac{p^{\prime}}{q^{\prime}}$.

Often rotation numbers $\rho_{F}(x)$ are considered $\bmod 1$, while rotation intervals $\operatorname{Rot}(F)$ need in general to be considered as real intervals since they can be quite long. However, since our interest in this paper is in quite short rotation intervals, 


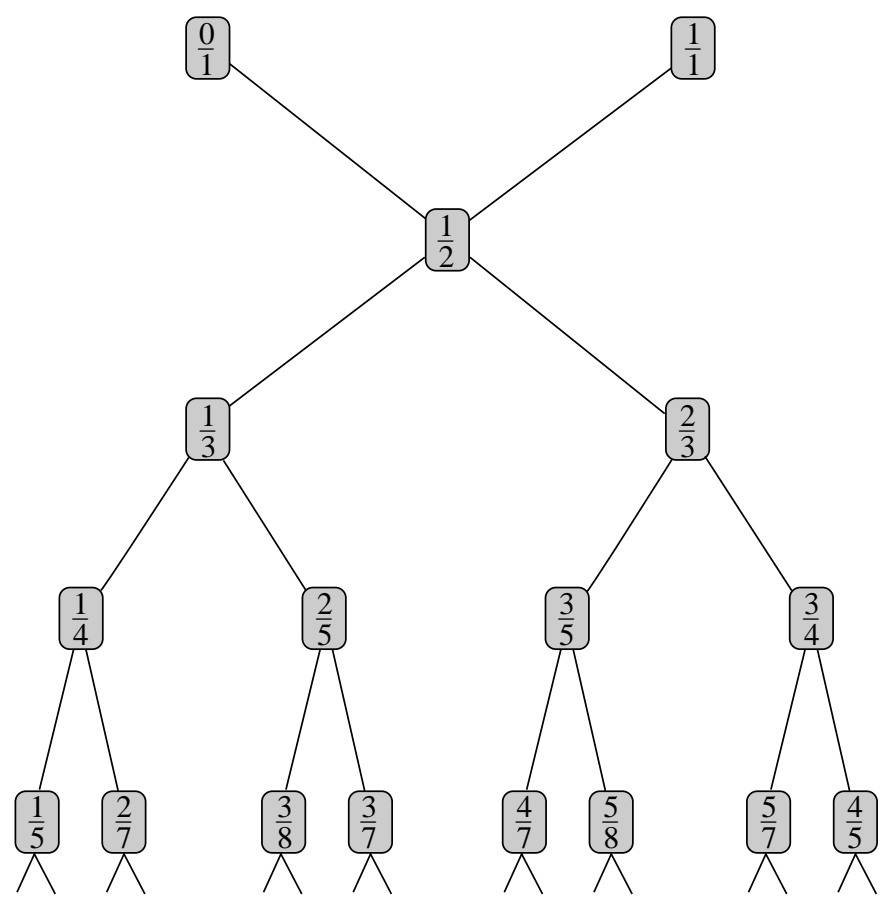

FIGURE 1

the reader may consider that all $\rho_{F}(x)$ 's are taken mod 1 for maps on which we focus our attention, although this does not allow us to explore the full parametrized families to which our maps belong.

Two-parameter families of smooth deformations of rotations such as the standard family defined by

$$
H_{(a, b)}(x)=x+a+\frac{b}{2 \pi} \cdot \sin (2 \pi x)
$$

are still the subject of much attention (see for instance the bibliography in [11], the recent universality results in [10], or the current literature on chaos in the natural sciences). Assume $F_{(a, b)}=F_{b}(x)+a$ is such a family where $F_{(a, 0)}$ is the rotation through angle $a$. The frequency-locking region with frequency $\omega, L_{\omega}$, is the set of parameters $(a, b)$ such that $\operatorname{Rot}\left(F_{(a, b)}\right)=\{\omega\}$. The height of $L_{\omega}$ is the largest value of $b$ such that some map $F_{(a, b)}$ belongs to $L_{\omega}$. It has recently been shown that for the family $\left\{H_{(a, b)}\right\}$ above, all frequency-locking regions are connected [5]. However, in Class $\mathcal{B}$ it is not known whether all frequency-locking regions are connected and pathologies as represented in Figure 2 have yet to be shown not to exist. Thus we call the connected component $L_{\omega}^{\mathbf{T}}$ of $L_{\omega}$ containing all invertible maps in the family $\left\{F_{(a, b)}\right\}$ the frequency-locking tongue with frequency $\omega$. The reduced height of $L_{\omega}$ is then the height of $L_{\omega}^{\mathbf{T}}$, i.e., the largest value of $b$ such that some map $F_{(a, b)}$ belongs to $L_{\omega}^{\mathbf{T}}$. Theorem 1 has the following corollary.

Corollary 1. In a smooth two-parameter family in Class $\mathcal{B}$ (as above), if $\frac{p}{q}$ is higher than $\frac{p^{\prime}}{q^{\prime}}$ in the Farey tree, then the reduced height of $L_{\frac{p}{q}}$ is greater than the reduced height of $L_{\frac{p^{\prime}}{q^{\prime}}}$. 


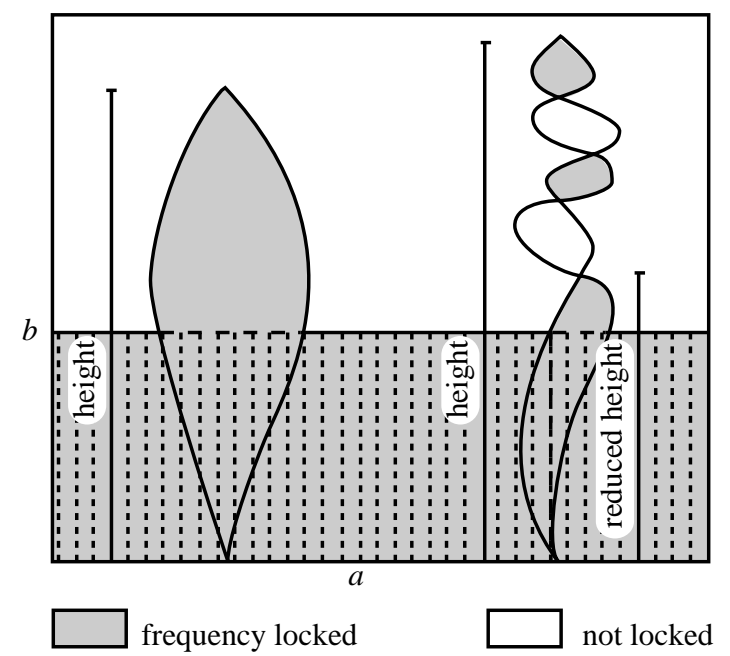

i.': diffeomorphisms

FiguRe 2

Remark 1. The fact that the inequality in the above corollary is strict follows from the proof of Theorem 1 (in the proof of Theorem 1 use that $A_{F}=M_{i}$ and $B_{F}=N_{i}$ at the tip of a tongue).

This corollary furthers our understanding of the boundary between a single rotation number and a nontrivial rotation interval in "nice" families. In the same setting, it was previously proved that this boundary is not locally connected $[2$, 6]. Notice that we deal with heights of frequency-locking regions; a much older problem is to determine whether the widths of these regions are organized along the Farey tree under some "reasonable" condition on a two-parameter family. The "classical" problem is, in fact, about isomorphisms of the circle (i.e., for instance, the case when $b<1$ in the standard family). This is why our title begins by "A" and not by "The".

\section{Preparatory material on Degree one lifts}

For the convenience of the reader and for completeness we begin with some basic facts about lift maps. These basic facts are used throughout this note. Again, there are many sources for the information in this section; for simplicity we continue to list [1].

Fact 1. Let $F$ be a degree-one lift. Then, $F_{k}^{n}(x)=F^{n}(x)+k n$, for all $x \in \mathbb{R}$, $k \in \mathbb{Z}$, and $n \in \mathbb{N}[1$, Proposition 3.1.7].

Fact 2. Let $F$ be a non-decreasing degree-one lift. Then for every $x \in \mathbb{R}, \underline{\rho}_{F}(x)=$ $\bar{\rho}_{F}(x)$ and this number is independent of $x[1$, Proposition 3.7.11]. Thus, Rot $(F)$ is a single point, which we will denote with $\rho(F)$.

Definition 1. Let $F$ be a degree-one lift, and let $x \in \mathbb{R}$. The lift-orbit of $x$ is defined to be $\operatorname{orb}(x, F)+\mathbb{Z}$, where $\operatorname{orb}(x, F)=\left\{x, F(x), F^{2}(x), \cdots\right\}$. We say that the lift-orbit of $x$ is a twisted lift-orbit provided that $F$ restricted to orb $(x, F)+\mathbb{Z}$ 
is non-decreasing. We say that $\operatorname{orb}(x, F)+\mathbb{Z}$ is a lifted cycle provided there is some $n \in \mathbb{N}^{*}$ such that $F^{n}(x)-x \in \mathbb{Z}$. The lift-orbit $\operatorname{orb}(x, F)+\mathbb{Z}$ is called a lifted $m$-cycle if $\operatorname{orb}(x, F)+\mathbb{Z}$ is a lifted cycle and if $m=\min \left\{n \in \mathbb{N}^{*} \mid F^{n}(x)-x \in \mathbb{Z}\right\}$. Lastly, orb $(x, F)+\mathbb{Z}$ is a twist-lifted $m$-cycle if $\operatorname{orb}(x, F)+\mathbb{Z}$ is a lifted $m$-cycle and a twisted lift-orbit (in this case we say that $x$ or $F$ has a twist-lifted $m$-cycle) [1, sections $3.2,3.3$ and 3.7$]$.

Fact 3. Let $F$ be a degree-one lift, and suppose that orb $(x, F)+\mathbb{Z}$ is a lifted $m$ cycle. Then, the cardinality of $(\operatorname{orb}(x, F)+\mathbb{Z}) \cap[y, y+1)=m$ for every $y \in \mathbb{R}[1$, Proposition 3.2.2].

Fact 4. If $F$ is a degree-one lift, orb $(x, F)+\mathbb{Z}$ a twist-lifted $m$-cycle, and $l \in \mathbb{Z}$ is such that $F^{m}(x)-x=l$, then $m, l$ are coprime and for every $y \in \operatorname{orb}(x, F)+\mathbb{Z}$ we have $\rho_{F}(y)=\frac{l}{m}[1$, Lemma 3.7.2 and Corollary 3.7.6].

Fact 5. Let $F$ be a degree-one lift, and let $x \in \mathbb{R}$ have a twist-lifted $q$-cycle with $F^{q}(x)-x=p$. Set $\operatorname{orb}(x, F)+\mathbb{Z}=\left\{\ldots, y_{-2}, y_{-1}, y_{0}, y_{1}, y_{2}, \ldots\right\}$ with $\cdots<y_{-2}<$ $y_{-1}<y_{0}<y_{1}<y_{2}<\cdots$. Then $F\left(y_{i}\right)=y_{i+p}$ for all $i \in \mathbb{Z}[1$, Lemma 3.7.4].

Lemma 1. Let $F, x, q, p$, and $\left\{y_{i}\right\}_{-\infty}^{\infty}$ be as in Fact 5 . Let $(\operatorname{orb}(x, F)+\mathbb{Z}) \cap[0,1)=$ $\left\{y_{0}, y_{1}, \cdots, y_{q-1}\right\}$; we can do this by Fact 3 . Then, $y_{i+p} \bmod 1=y_{(i+p) \bmod q}$ for each $i \in \mathbb{Z}$.

Proof. Fix $i \in \mathbb{Z}$. Choose the unique $m \in \mathbb{Z}$ such that $i+p \in\{m q, m q+1, \ldots$, $(m+1) q-1\}$; we can do this since $\{m q+j \mid m \in \mathbb{Z}$ and $0 \leq j \leq q-1\}=\mathbb{Z}$. Say $i+p=m q+t$ with $0 \leq t \leq q-1$. Then $(i+p) \bmod q=t$. Hence, if we show that $y_{m q+t} \bmod 1=y_{t}$, then we are done. But $\operatorname{orb}(x, F)+\mathbb{Z}=(\operatorname{orb}(x, F)+\mathbb{Z})+\mathbb{Z}$ and Fact 3 imply that $y_{i}+m q=y_{m q+i}$ for $0 \leq i \leq q-1$. Thus, $y_{m q+t} \bmod 1=$ $\left(y_{t}+m q\right) \bmod 1=y_{t}$. This completes the proof.

Definition 2. Let $F$ be a degree-one lift. The maps $F_{l}$ and $F_{u}$ are defined as follows:

$$
\begin{aligned}
& F_{l}(x)=\inf \{F(y) \mid y \geq x\}, \\
& F_{u}(x)=\sup \{F(y) \mid y \leq x\}
\end{aligned}
$$

For $0 \leq \mu \leq \mu_{1}=\sup _{x \in \mathbb{R}}\left(F-F_{l}\right)(x)$, the map $F_{\mu}$ is defined by

$$
F_{\mu}=\left(\min \left(F, F_{l}+\mu\right)\right)_{u}
$$

Each of $F_{l}, F_{u}$, and $F_{\mu}$ are non-decreasing degree-one lifts (see Figure 3 and [1, Section 3.7]).

Fact 6. The function $F \mapsto \rho(F)$ is continuous on the space of all non-decreasing degree-one lifts [1, Lemma 3.7.12].

Fact 7. Let $F$ and $G$ be degree-one lifts with at least one of the lifts non-decreasing. If $F \leq G$, then $F^{n} \leq G^{n}$ for all $n \in \mathbb{N}[1$, Lemma 3.7.19]. 


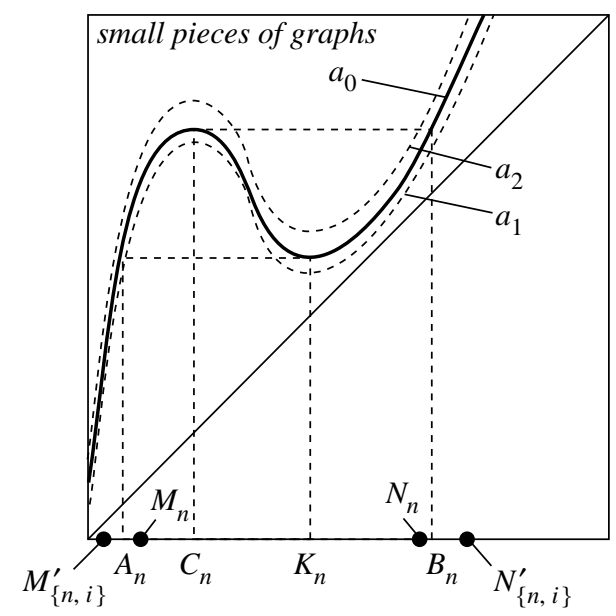

FiguRE 3

Fact 8 [1, Theorem 3.7.20 and proof $]$. Let $F$ be a degree-one lift. Then the following hold.

(i) $\operatorname{Rot}(F)=\left[\rho\left(F_{l}\right), \rho\left(F_{u}\right)\right]$.

(ii) If a is a rational number in $\operatorname{Rot}(F)$, then there exists a twist-lifted cycle of $F$ with rotation number $a$ and disjoint from Const $(F)$. Here, Const $(F)$ is the maximal open set on which $F$ is locally constant.

(iii) Recall the family $\left\{F_{\mu}\right\}_{\mu \in\left[0, \mu_{1}\right]}$ from Definition 2. For each a $\in$ $\left[\rho\left(F_{l}\right), \rho\left(F_{u}\right)\right]$, there exists $\mu_{a} \in\left[0, \mu_{1}\right]$ with $\rho\left(F_{\mu_{a}}\right)=a$.

Fact $9\left[11\right.$, Proposition 4.2]. Let $F$ be in Class $\mathcal{B}$ and $\frac{p}{q} \in \operatorname{Rot}(F)$. Then $F$ has at most two twist-lifted cycles with rotation number $\frac{p}{q}$.

Remark 2. Let $F$ be a degree-one lift, and consider the one-parameter family $\left\{F_{a}\right\}$ with $a \in \mathbb{R}$. For every $a \in \mathbb{R}$, we have that $\left(F_{a}\right)_{l}=F_{l}+a$ and $\left(F_{a}\right)_{u}=F_{u}+a$. From this and Fact 7 we see that if $a_{1}<a_{2}$, then $\rho\left(\left(F_{a_{1}}\right)_{l}\right) \leq \rho\left(\left(F_{a_{2}}\right)_{l}\right)$ and $\rho\left(\left(F_{a_{1}}\right)_{u}\right) \leq \rho\left(\left(F_{a_{2}}\right)_{u}\right)$. Thus (recall Fact $\left.8(\mathrm{i})\right)$, if $a, b \in \mathbb{R}$ are such that both $F_{a}$ and $F_{b}$ are frequency locked with $\rho\left(F_{a}\right)>\rho\left(F_{b}\right)$, then $a>b$.

Remark 3. From Fact 1 and the beginning of Remark 2, we have that $\rho\left(\left(F_{k}\right)_{l}\right)=$ $\rho\left(F_{l}\right)+k$ and $\rho\left(\left(F_{k}\right)_{u}\right)=\rho\left(F_{u}\right)+k$ for each $k \in \mathbb{Z}$.

\section{Preparatory material From number theOry}

The material in this section is not new; however, we recall it in a form which meets our needs. Lemmas 2 and 3 are simply technical lemmas needed for the proof of Lemma 4 of Section 4 . Lemma 4 is essential to our proof of Theorem 1. Lemma 3 is proven similar to Lemma 2 .

Lemma 2. Let $\frac{u}{v}=\frac{p_{1}}{q_{1}} \oplus \frac{p}{q}$, i.e., $\frac{u}{v}$ is the left child of $\frac{p}{q}$. Then

(i) $u q-p v=-1$,

(ii) $p_{1} q-p q_{1}=-1$,

(iii) if $i_{0} \in\{0,1, \cdots, q-2\}$, then $p_{1} q+1 \leq i_{0}+q_{1} p \leq p_{1} q+(q-1)$, and

(iv) if $i_{0} \in\{0,1, \cdots, q-2\}$, then $\left(i_{0}+q_{1} p\right) \bmod q=i_{0}+1$. 
Proof. Statement (i) is easily obtained from [9, p. 23, Theorem 28]. From part (i), $v=q_{1}+q$, and $u=p_{1}+p$, we have that $-1=u q-p v=\left(p_{1}+p\right) q-p\left(q_{1}+q\right)$ and thus (ii) holds.

To see (iii) notice that $0 \leq i_{0} \leq q-2$ gives that $q_{1} p \leq i_{0}+q_{1} p \leq(q-2)+q_{1} p$. But by part (ii), $q_{1} p=p_{1} q+1$; therefore, $p_{1} q+1 \leq i_{0}+q_{1} p \leq p_{1} q+(q-1)$ and (iii) holds. For part (iv) use that $i_{0}+q_{1} p=i_{0}+p_{1} q+1$ (by part (ii)) and $1 \leq i_{0}+1 \leq q-1$ to conclude that $\left[\left(i_{0}+q_{1} p\right)=\left(i_{0}+1+p_{1} q\right)\right] \bmod q=i_{0}+1$.

Lemma 3. Let $\frac{a}{b}=\frac{p}{q} \oplus \frac{p_{2}}{q_{2}}$, i.e., $\frac{a}{b}$ is the right child of $\frac{p}{q}$. We have

(i) $a q-p b=1$,

(ii) $p q_{2}-p_{2} q=-1$,

(iii) if $1 \leq i_{0} \leq q-1$, then $p_{2} q \leq i_{0}+q_{2} p \leq p_{2} q+(q-2)$, and

(iv) if $1 \leq i_{0} \leq q-1$, then $\left(i_{0}+q_{2} p\right) \bmod q=i_{0}-1$.

\section{Proof of the main theorem}

In the interest of simplifying the proof of Theorem 1, we begin with three lemmas. These lemmas are essentially well known, but we include them here for the convenience of the reader. Lemma 4 is just a small remark from the more general theory of coding problems in dynamics and in number theory. For information about this more general theory see for example $[7,8,13]$. Lemma 5 can be obtained, for example, from the work in $[3,11]$, and Lemma 6 from $[3,4,11]$. Notation in this section is as in previous sections.

Lemma 4. Let $F$ be a degree-one lift, and let $x \in \mathbb{R}$ have a twist-lifted $q$-cycle with $F^{q}(x)-x=p$. Set $\operatorname{orb}(x, F)+\mathbb{Z}=\left\{\ldots, y_{-2}, y_{-1}, y_{0}, y_{1}, y_{2}, \ldots\right\}$ with $\cdots<y_{-2}<$ $y_{-1}<y_{0}<y_{1}<y_{2}<\cdots$ and with $(\operatorname{orb}(x, F)+\mathbb{Z}) \cap[0,1)=\left\{y_{0}, y_{1}, \ldots, y_{q-1}\right\}$. For emphasis recall that $\frac{p}{q}=\frac{p_{1}}{q_{1}} \oplus \frac{p_{2}}{q_{2}}$. Lastly, let $i_{0} \in\{0,1, \ldots, q-2\}$ and $i_{1} \in$ $\{1,2, \ldots, q-1\}$. Then we have that

(i) $F^{q_{1}}\left(y_{i_{0}}\right) \bmod 1=y_{i_{0}+1}$,

(ii) $F^{q_{1}}\left(y_{i_{0}}\right) \in\left[p_{1}, p_{1}+1\right)$,

(iii) $F^{q_{2}}\left(y_{i_{1}}\right) \bmod 1=y_{i_{1}-1}$, and

(iv) $F^{q_{2}}\left(y_{i_{1}}\right) \in\left[p_{2}, p_{2}+1\right)$.

Proof. We first prove statements (i) and (ii). By Fact $5, F^{q_{1}}\left(y_{i_{0}}\right)=y_{i_{0}+q_{1} p}$ and therefore $\left[F^{q_{1}}\left(y_{i_{0}}\right)=y_{i_{0}+q_{1} p}\right] \bmod 1=y_{\left(i_{0}+q_{1} p\right) \bmod q}$, by Lemma 1. However, $\left(i_{0}+q_{1} p\right) \bmod q=i_{0}+1$ by Lemma 2 (iv) and hence (i) holds. To see (ii) first note that $p_{1} q+1 \leq i_{0}+q_{1} p \leq p_{1} q+(q-1)$ (by Lemma 2 (iii)) and then use Fact 3 along with $F^{q_{1}}\left(y_{i_{0}}\right)=y_{i_{0}+q_{1} p}$ (Fact 5$)$.

Statements (iii) and (iv) are proven in a similar manner except that now Lemma 3 is used in place of Lemma 2.

Remark 4. Let $F$ be a degree-one lift in Class $\mathcal{A}$. Then there exists a unique point $A_{F}$ such that $K_{F}-1<A_{F}<C_{F}$ and $F\left(A_{F}\right)=F\left(K_{F}\right)$; similarly there is a unique point $B_{F}$ such that $K_{F}<B_{F}<C_{F}+1$ and $F\left(B_{F}\right)=F\left(C_{F}\right)$ (see Figure 4). Clearly, the origin can be chosen so that $0 \leq A_{F}<C_{F}<K_{F}<B_{F}<1$. Also notice that for a one-parameter family of the form $\left\{F_{a}\right\}$ with $a \in \mathbb{R}$, all points $A_{F_{a}}$, $C_{F_{a}}, K_{F_{a}}$, and $B_{F_{a}}$ are independent of the parameter $a$, i.e., $A_{F_{a}}=A_{F}, C_{F_{a}}=C_{F}$, $K_{F_{a}}=K_{F}$, and $B_{F_{a}}=B_{F}$ for all $a \in \mathbb{R}$. Thus, throughout the rest of this note we use simply $A_{F}, C_{F}, K_{F}$, and $B_{F}$. 


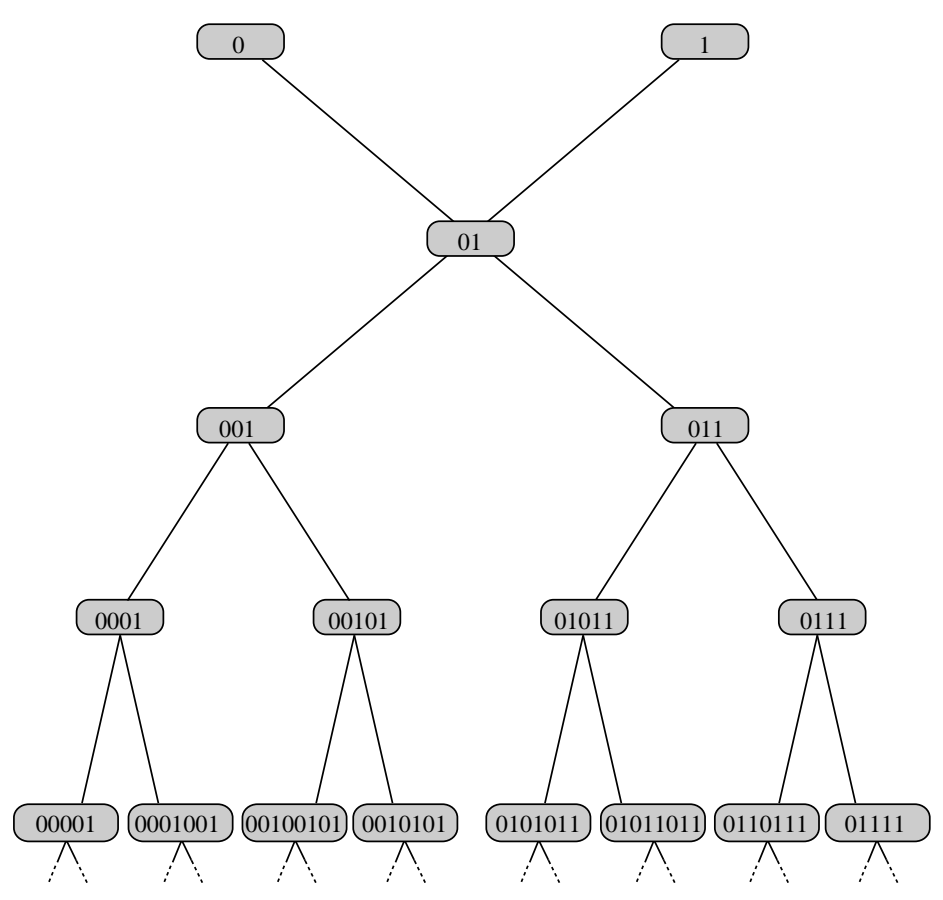

FiguRE 4

Lemma 5. Let $F$ be a degree-one lift in Class $\mathcal{A}$. Let $l \in \mathbb{Z}$ and $m \in \mathbb{N}$ be coprime. Assume that no $F_{a}$, for $a \in \mathbb{R}$, is frequency locked with frequency $\frac{l}{m}$. Then for some $a_{0} \in \mathbb{R}, F_{a_{0}}$ has a twist-lifted $m$-cycle with rotation number $\frac{l}{m}$ which has two consecutive points $M<N$ with $M \in\left(A_{F}, C_{F}\right)$ and $N \in\left(K_{F}, B_{F}\right)$ such that $F_{a_{0}}(M)<F_{a_{0}}(N)$.

Proof. We first show that there is some $\hat{a} \in \mathbb{R}$ with $\rho\left(\left(F_{\hat{a}}\right)_{l}\right)=\frac{l}{m}$. But this follows immediately from $\rho\left(\left(F_{k}\right)_{l}\right)=\rho\left(F_{l}\right)+k$ for all $k \in \mathbb{Z}$ (Remark 3) and the function $G \mapsto \rho(G)$ being continuous on all non-decreasing degree-one lifts (Fact 6 ). Hence, choose $\hat{a} \in \mathbb{R}$ such that $\rho\left(\left(F_{\hat{a}}\right)_{l}\right)=\frac{l}{m}$.

Next we show there exists $a_{0} \in \mathbb{R}$ such that $\frac{l}{m}$ is in the interior of the closed interval $\operatorname{Rot}\left(F_{a_{0}}\right)$. There exist parameters $a<\hat{a}$ such that $\rho\left(\left(F_{a}\right)_{l}\right)<\rho\left(\left(F_{\hat{a}}\right)_{l}\right)=\frac{l}{m}$ since $\rho\left(\left(F_{\hat{a}-1}\right)_{l}\right)=\rho\left(\left(F_{\hat{a}}\right)_{l}\right)-1=\frac{l}{m}-1$ by Remark 3. Now the existence of $a_{0}$ follows from the continuity of $F \mapsto \rho(F)$ on the non-decreasing degree-one lifts (Fact 6 ) and the assumption that no $F_{a}$ is frequency locked with frequency $\frac{l}{m}$.

Lastly we show that this $a_{0}$ satisfies our lemma. Set $G=F_{a_{0}}$. Apply Fact 8(iii) to the family $\left\{G_{\mu}\right\}$ to choose a value $\mu$ so that $\rho\left(G_{\mu}\right)=\frac{l}{m}$. Next apply Fact 8(ii) to get a twist-lift $m$-cycle of $G_{\mu}$ with rotation number $\frac{l}{m}$ and disjoint from $\operatorname{Const}\left(G_{\mu}\right)$; let $\mathcal{O}$ denote this twist-lift $m$-cycle. First notice that the construction of $G_{\mu}$ and the fact that $\mathcal{O}$ does not intersect $\operatorname{Const}\left(G_{\mu}\right)$ give that $\mathcal{O}$ is a twist-lift $m$ cycle of $G=F_{a_{0}}$. Moreover, $\mathcal{O} \cap[0,1)$ does not intersect the interval $\left(C_{G}, K_{G}\right)$ since $\mathcal{O} \cap \operatorname{Const}\left(G_{\mu}\right)=\emptyset$. Also, both $\mathcal{O} \cap\left[0, C_{G}\right]$ and $\mathcal{O} \cap\left[K_{G}, 1\right)$ are non-empty since $G$ is strictly increasing on both of $\left[0, C_{G}\right]$ and $\left[K_{G}, 1\right)$. Let $M=\max \left\{y \mid y \in \mathcal{O} \cap\left[0, C_{G}\right]\right\}$, and let $N=\min \left\{y \mid y \in \mathcal{O} \cap\left[K_{G}, 1\right)\right\}$. Then, $G(M)<G(N)$ by construction of 
$G_{\mu}$. If $M<A_{G}$ or $N>B_{G}$, then $\rho\left(G_{l}\right)=\frac{l}{m}$ or $\rho\left(G_{u}\right)=\frac{l}{m}$; but this contradicts our choice of $a_{0}$ (namely that $\frac{l}{m}$ is in the interior of $\operatorname{Rot}(G)=\left[\rho\left(G_{l}\right), \rho\left(G_{u}\right)\right]$ ).

Lemma 6. Let $F$ be a degree-one lift in Class $\mathcal{B}$. Let $l \in \mathbb{Z}$, and let $m \in \mathbb{N}$ be coprime. Assume that $F_{a}$ is frequency locked with frequency $\frac{l}{m}$, for some fixed $a \in \mathbb{R}$. Then, $F_{a}$ has at least one twist-lifted $m$-cycle with rotation number $\frac{l}{m}$ and with two consecutive points $M<N$ such that $M \in\left[0, A_{F}\right]$ and $N \in\left[B_{F}, 1\right)$.

Proof. The fact that $F_{a}$ has at least one twist-lifted $m$-cycle with rotation number $\frac{l}{m}$ is immediate from Fact 8(ii). However, by Fact $9, F_{a}$ has at most two such twist-lifted cycles. Set $F_{a}=G$, and consider the family $\left\{G_{\mu}\right\}$ with $\mu \in\left[0, \mu_{1}\right]$ from Definition 2. Now, $\operatorname{Rot}(G)=\frac{l}{m}$ implies that $\rho\left(G_{\mu}\right)=\frac{l}{m}$ for all $\mu \in\left[0, \mu_{1}\right]$; see Fact 7. Hence (see Fact 8(ii)), each $G_{\mu}$ has a twist-lifted $m$-cycle with rotation number $\frac{l}{m}$ which is disjoint from $\operatorname{Const}\left(G_{\mu}\right)$. However, each of these twist-lifted cycles are also twist lifted-cycles for $G$ (recall the construction of $G_{\mu}$ and then use that each such twist-lifted cycle is disjoint from $\left.\operatorname{Const}\left(G_{\mu}\right)\right)$. The lemma now follows, for otherwise using these twist-lifted cycles from the $\left\{G_{\mu}\right\}$, we would have that $G=F_{a}$ has infinitely many twist-lifted $m$-cycles, a contradiction.

Proof of Theorem 1. With the notation of Theorem 1, assume that no $F_{a}$ is frequency locked with frequency $\frac{p}{q}$. Then by Lemma 5 , some $F_{a_{0}}$ has a twist-lifted $q$-cycle with rotation number $\frac{p}{q}$ and with two consecutive points $M<N$ such that

$$
M \in\left(A_{F}, C_{F}\right), N \in\left(K_{F}, B_{F}\right), \text { and } F_{a_{0}}(M)<F_{a_{0}}(N) .
$$

To prove the theorem, it is enough to consider the cases when $\frac{p^{\prime}}{q^{\prime}}$ is a child of $\frac{p}{q}$. Hence, we assume that either $\frac{p^{\prime}}{q^{\prime}}=l\left(\frac{p}{q}\right)$ or that $\frac{p^{\prime}}{q^{\prime}}=r\left(\frac{p}{q}\right)$.

Assume ad absurdum that there exists $a_{1} \in \mathbb{R}$ such that $F_{a_{1}}$ is frequency locked with frequency $l\left(\frac{p}{q}\right)=\frac{p_{1}}{q_{1}} \oplus \frac{p}{q}$, or $a_{2} \in \mathbb{R}$ such that $F_{a_{2}}$ is frequency locked with frequency $r\left(\frac{p}{q}\right)=\frac{p}{q} \oplus \frac{p_{2}}{q_{2}}$. From Remark 2 we deduce that

$$
a_{1}<a_{0}<a_{2} .
$$

By Lemma 6 , each of $F_{a_{1}}$ and $F_{a_{2}}$ admit a twisted lift cycle with rotation number $l\left(\frac{p}{q}\right)$ and $r\left(\frac{p}{q}\right)$ respectively and with consecutive points $M_{1}<N_{1}$ and $M_{2}<N_{2}$ respectively such that

$$
M_{i} \in\left[0, A_{F}\right] \text { and } N_{i} \in\left[B_{F}, 1\right)
$$

for $i=1,2$. Hence,

$$
M_{i} \leq A_{F}<M<C_{F}<K_{F}<N<B_{F} \leq N_{i}
$$

for $i=1,2$. From (2), (4), and the fact that none of the twist-lifted cycles explore the regions where the maps are decreasing, and using the consecutive property of the points $M, N$ and $M_{i}, N_{i}$, we deduce that

$$
F_{a_{1}}^{m}\left(M_{1}\right) \leq F_{a_{0}}^{m}(M) \text { and } F_{a_{2}}^{m}\left(N_{2}\right) \geq F_{a_{0}}^{m}(N),
$$

for every $m \in \mathbb{N}$. 
From Lemma 4(i), (ii) applied to $\frac{p}{q}=\frac{p_{1}}{q_{1}} \oplus \frac{p_{2}}{q_{2}}$ and to $l\left(\frac{p}{q}\right)=\frac{p_{1}}{q_{1}} \oplus \frac{p}{q}$ respectively we have that

$$
F_{a_{0}}^{q_{1}}(M) \in\left[p_{1}, p_{1}+1\right) \text { with } F_{a_{0}}^{q_{1}}(M) \bmod 1=N
$$

and

$$
F_{a_{1}}^{q_{1}}\left(M_{1}\right) \in\left[p_{1}, p_{1}+1\right) \text { with } F_{a_{1}}^{q_{1}}\left(M_{1}\right) \bmod 1=N_{1}
$$

Similarly, Lemma 4(iii), (iv) applied to $\frac{p}{q}=\frac{p_{1}}{q_{1}} \oplus \frac{p_{2}}{q_{2}}$ and $r\left(\frac{p}{q}\right)=\frac{p}{q} \oplus \frac{p_{2}}{q_{2}}$ respectively give that

$$
F_{a_{0}}^{q_{2}}(N) \in\left[p_{2}, p_{2}+1\right) \text { with } F_{a_{0}}^{q_{2}}(N) \bmod 1=M
$$

and

$$
F_{a_{2}}^{q_{2}}\left(N_{2}\right) \in\left[p_{2}, p_{2}+1\right) \text { with } F_{a_{2}}^{q_{2}}\left(N_{2}\right) \bmod 1=M_{2}
$$

However (4), (6), (7) and (4), (8), (9) give respectively that

$$
F_{a_{0}}^{q_{1}}(M)<F_{a_{1}}^{q_{1}}\left(M_{1}\right)
$$

and

$$
F_{a_{0}}^{q_{2}}(N)>F_{a_{2}}^{q_{2}}\left(N_{2}\right)
$$

Lastly, both (10) and (11) contradict (5) (with $m=q_{1}$ and $m=q_{2}$ respectively in (5)). This completes the proof of Theorem 1.

\section{REFERENCES}

1. L. Alsedá, J. Llibre, and M. Misiurewicz, Combinatorial dynamics and entropy in dimension one, Adv. Ser. Nonlinear Dynam., vol. 5, World Scientific, River Edge, NJ, 1993. CMP 94:06

2. P. M. Blecher and M. V. Jakobson, Absolutely continuous invariant measures for some maps of the circle, Statistical Physics and Dynamical Systems, Progr. Phys., vol. 10, Birkhäuser, Boston, Basel, and Stuttgart, 1985, pp. 303-315. MR 87f:58081

3. P. L. Boyland, Bifurcations of circle maps: Arnol'd tongues, bistability and rotation intervals, Comm. Math. Phys. 106 (1986), 353-381. MR 88c:58045

4. A. Chenciner, J. M. Gambaudo, and C. Tresser, Une remarque sur la structure des endomorphismes de degré un du cercle, C. R. Acad. Sci. Paris Sér. I Math 299 (1984), 771-773. MR 86g:58081

5. A. Epstein, L. Keen, and C. Tresser, The set of maps $f_{a, b}: \theta \mapsto \theta+a+\frac{b}{2 \pi} \cdot \sin (2 \pi \theta)$ with any given rotation numbers is contractible, Comm. Math. Phys. (to appear).

6. B. Friedman and C. Tresser, Comb structure in hairy boundaries: some transition problems for circle maps, Phys. Lett. A 117 (1986), 15-22. MR 87j:58061

7. J. M. Gambaudo and C. Tresser, On the dynamics of quasi-contractions, Bol. Soc. Brasil. Mat. (N.S.) 19 (1986), 61-114. MR 91d:54033

8. L. Goldberg and C. Tresser, Rotation orbits and the farey tree, Ergodic Theory Dynamical Systems (to appear).

9. G. H. Hardy and E. M. Wright, An introduction to the theory of numbers, Clarendon Press, Oxford, 1979. MR 81i:10002 
10. K. M. Khanin, Universal estimates for critical circle mappings, Chaos 1 (1991), 181-186. MR 93a: 58106

11. R. S. Mackay and C. Tresser, Transition to topological chaos for circle maps, Phys. D 19 (1986), 206-237. MR 87k:58182

12. H. Poincaré, Sur les courbes définies par des équations différentielles, J. Math. Pures Appl. (4) 1 (1885), 167-244; Euvres Complètes, Vol. 1, Gauthier-Villars, Paris, 1951.

13. R. M. Siegel, C. Tresser, and G. Zettler, A decoding problem in dynamics and in number theory, Chaos 2 (1982), 473-494.

14. D. J. Uherka, C. Tresser, R. Galeeva, and D. K. Campbell, Solvable models for the quasiperiodic transition to chaos, Phys. Lett. A. 170 (1992), 189-194; Chaos (to appear). MR 94e:58092

Department of Mathematical Sciences, University of Wisconsin, Milwaukee, WisconSIN 53211

E-mail address: kmbrucks@csd.uwm.edu

Thomas J. Watson Research Center, I.B.M., P.O. Box 218, Yorktown Heights, New YORK 10598

E-mail address: tresser@watson.ibm.com 